\title{
INCREASE OF CAPACITY ON THE SHINKANSEN HIGH-SPEED LINE USING VIRTUAL COUPLING
}

\author{
TILO SCHUMANN \\ DLR Institute of Transportation Systems, Braunschweig, Germany
}

\begin{abstract}
The coupling and decoupling of trains while they are in motion is one of the research fields of the DLR project, Next Generation Train. The coupling will be non-mechanical, but virtual and will be maintained by means of distance control technology. A special challenge of the virtual coupling in railway operation is the peril point of the switch in case of failure during the passage of two trains which are coming from different tracks, running in close succession at high speed. Therefore some concepts are unrealizable with the current switch technology. Nevertheless there are situations where virtual coupling seems promising.

One scenario is the virtual coupling at low speed, for example in the proximity of a station, where a safe brake is possible in case of a switch failure. The 'Shinkansen' scenario aims to increase line capacity on the Tokaido high-speed line in Japan between Tokio and Osaka. The fastest 'Nozomi' trains are stopping only at major stations. These stations have two tracks per direction with a 400-metre platform. The idea is to double the number of Nozomi trains. So in every stopping station, there are two trains arriving and departing in a short interval. The distance on the open line is not that close, so it is more a ride within relative braking distance than a virtual coupling.

To evaluate that scenario a special railway operation simulation has been developed, which is able to handle virtual coupling. The simulation shows the possibility to increase seating capacity from 15,000 to 23,000 seats per hour and direction. One bottleneck is occurring in Nagoya, where an additional platform is needed. Also the return time in Tokio has to be shortened to no more than $15 \mathrm{~min}$.

Keywords: Capacity increase, Next Generation Train, Shinkansen, virtual coupling.
\end{abstract}

\section{INTRODUCTION}

Splitting and coupling of trains is currently done while stopping at stations. The idea to move this action to the line promises some benefits compared to the current situation.

Today coupling and splitting enables a more intensive use of the network because two trains merge into one and therefore have less capacity requirements. This is because the track capacity is primarily restricted by the number of trains but not by the length of trains. In addition less operating staff is needed and the vehicle utilization gets more efficient. Compared to a feeder line system train coupling provides a convenient travel for the passengers without the need to change trains. The only challenge for the passenger is to be in the right section before the train is split up.

One handicap is the coupling procedure. Due to the mechanical character technical failures may occur and the synchronization of trains is challenging in a system in case of delays. To cope with this, larger buffer times are planned, resulting in negative effects on the overall travel time.

The present article discusses operational scenarios using 'virtual coupling', which means that the coupling is not done mechanically anymore. The close distance between two trains, which could be, for example $10 \mathrm{~m}$, is maintained with localization and communication technology.

The virtual coupling is part of the research project Next Generation Train (NGT) of the German Aerospace Center (DLR) [1] (Fig. 1). 


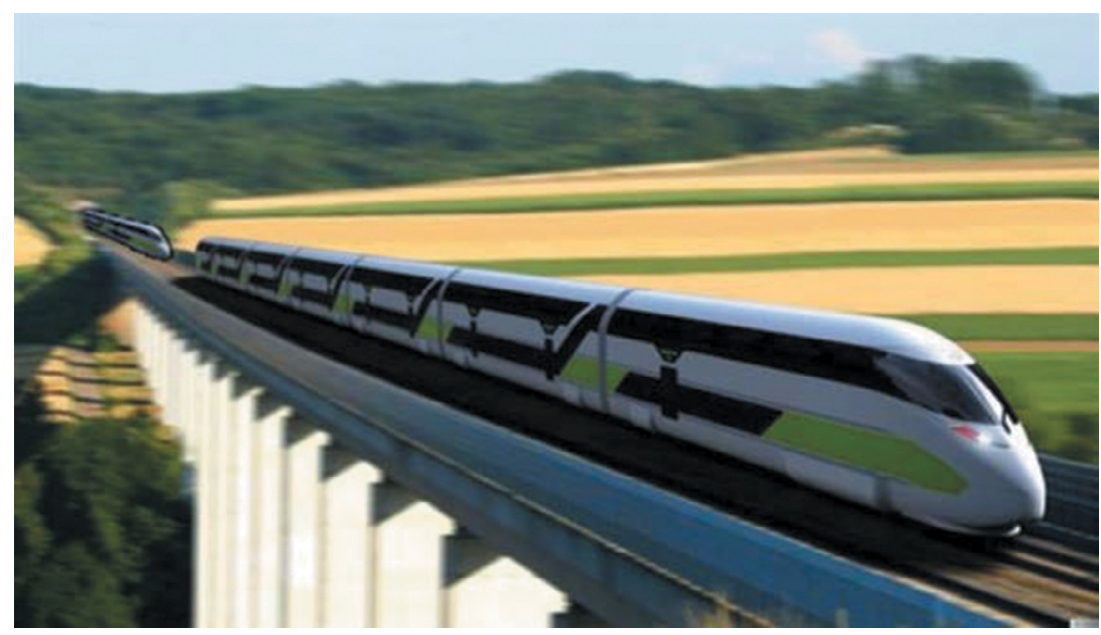

Figure 1: Impression of the virtual coupling of two NGT link trains.

\section{VIRTUAL COUPLING OPERATIONAL SCENARIOS}

The switch is one very important constraint for the development of operational scenarios. This is due to the fact that the switching may fail and the next train has to be able to stop before passing the switch [2]. With this constraint the benefit of virtual coupling is limited. The following discussion distinguishes between two switch technologies:

- Network with conventional switches

- Network with passive switches at junctions

Passive switches allow the trains to select their way themselves. There are no movable parts which could fail. Unfortunately there are no practical solutions for passive switches for highspeed traffic.

\subsection{Scenarios with conventional switches}

\subsubsection{Slip Coaching}

The Slip Coaching Scenario has its origin in the 19th century in the United Kingdom [3]. At that time the carriages at the end of a train were uncoupled from the rest in order to stop at a station. The main part of the train continued the journey without loss of time. A disadvantage is the effort for shunting and the coupling to the opposite train.

With electric multiple units this procedure becomes easier because there are no shunting locomotives necessary and also the coupling is possible. For this the slip train part has to accelerate ahead of the non-stopping part of the train. For safety reasons the accelerating train has to start early and travel a longer part alone, so that a virtual coupling manoeuvre can be done without violating the braking distance section in front of the non-stopping part.

This scenario is not investigated deeper yet, but there are possible applications within the German ICE network. For example smaller cities like Göttingen, Kassel or Fulda are stations where the passenger volume is lower and the percentage of transients is very high. A slip procedure is likely to reduce travel time. 


\subsection{2 'Shinkansen' Scenario}

This is the scenario which this article especially focuses on. It is named after the Japanese high-speed network, because this is an obvious application area for the procedure. The initial situation is a railway line which is heavily utilized by homogeneous traffic. There is the necessity for extra capacity especially on the section Tokio-Osaka, wherefore the ChuoShinkansen (with maglev technology) is planned.

The idea is to increase the capacity with virtual coupling. To avoid the switch problem, the coupling happens close to the stopping stations where the speed is lower and therefore the braking distances are shorter. When the first train passed the common switch, the second train starts and enters the occupied block and respectively follows the first train in a relative braking distance which also can be the virtual coupling (if both are running the same speed).

Additionally the stopping time for the Kodama trains could be reduced, if two overtaking trains are virtually coupled (see Fig. 2).

\subsection{Scenarios with passive switches}

\subsubsection{Coupling and splitting at junctions}

This is the 'classic' scenario considering the virtual coupling. Two trains arrive from different lines and pass a switch at a junction within a short time interval. Then they are coupling virtually and continue their journey together. Later they split ahead of the dividing switch and continue their journey on different lines (see Fig. 3).

The challenges for this scenario are the creation of a switch which can be passed safely in any situation and state and the exact coordination of speed and position of the trains. A difficult case is a loss of speed of the designated first train shortly before passing the switch. This could result in a side-on collision, so the speed of the second train has already to be regulated before the coupling procedure.

Since there are no passive switches available for high-speed traffic this scenario is not further investigated here.

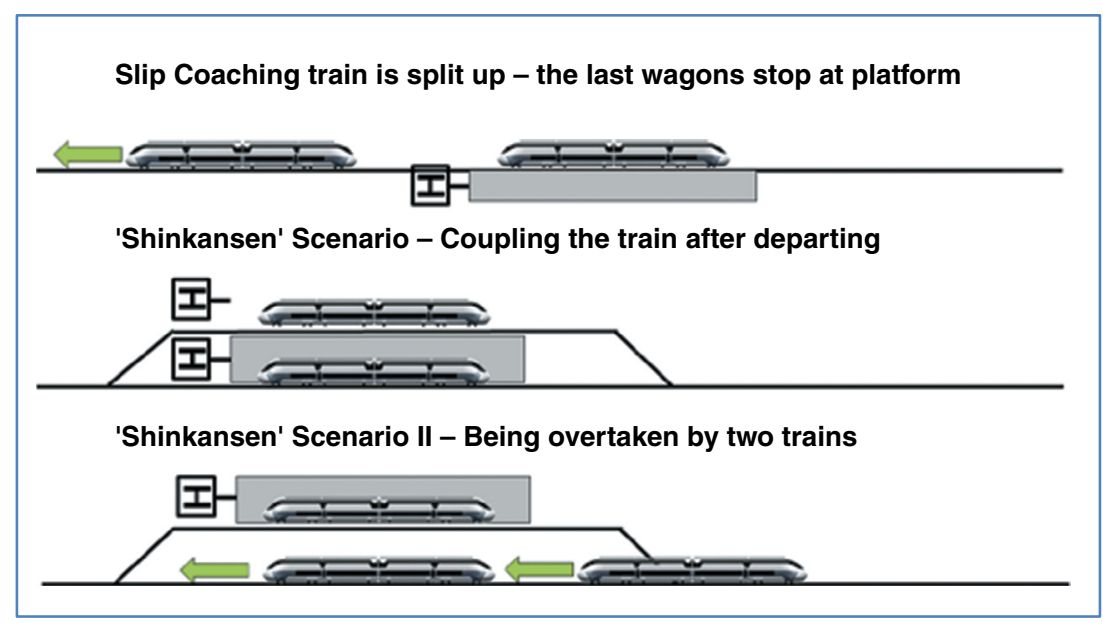

Figure 2: Schematics of virtual coupling scenarios with conventional switch. 


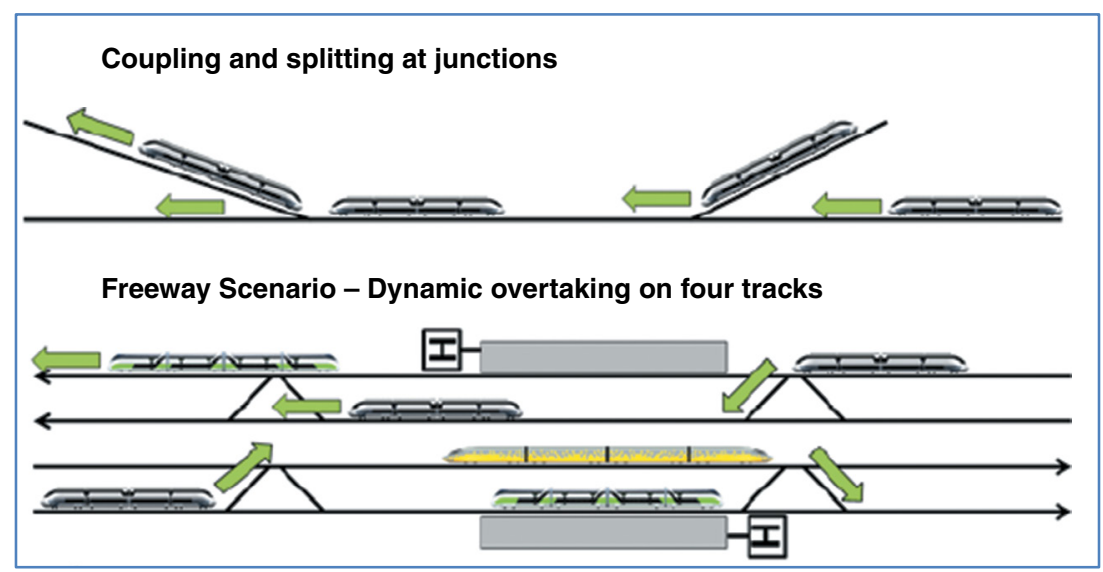

Figure 3: Schematics with virtual coupling scenarios with a new type of switch.

\subsection{2 'Highway' Scenario}

The highway scenario is similar to the coupling-and-splitting scenario; however the trains are not coupling virtually but following each other at least with the relative braking distance. They are not trying to reduce the distance in such a way that they reach the 'coupled state'. This would lead to a longer travel time for the leading train because of lowering the speed.

\subsection{3 'Freeway' Scenario}

The freeway scenario is the 'most fictional' of all scenarios. The eponym is the road-type freeway, where there are at least two lanes per direction. This is also the goal for the railway line, which has at least four tracks for both directions. There are connecting switches in regular distance to allow overtaking of slower trains or getting away from the fast lane to give the way for faster trains (see Fig. 3). Basically this is already realized on some railway lines in the world with the conventional signalling technology, but with the use of passive switches, virtual coupling and travelling within a relative braking distance the benefits are becoming even more significant. This scenario is also not investigated further.

\section{SIMULATION TOOL 'DFSIMU'}

To evaluate the scenarios it is not possible to test the procedures in reality, but they can be simulated first. Conventional simulation tools like OpenTrack [4], RailSys [5] or FBS [6] are not able to handle virtual coupling or trains runs at relative braking distance. Some can handle moving block, but this allows only running with an absolute braking distance.

The tool DFSimu was created by DLR in order to simulate railway operations with virtual coupling or at least travelling within a relative braking distance.

The tool is written in Python and consists of different modules for

- data handling,

- simulation and driving dynamics calculation,

- conventional signalling,

- coupling and splitting,

- infrastructure management,

- train type management,

- timetable management, 
- train management,

- protocolling and result plotting,

- OpenStreetMap import.

The traffic of the Tokaido Shinkansen with $515 \mathrm{~km}$ of line length, 66 trains with 15,700 train-kilometres and the time between 16:00 and 21:00 can be simulated on a conventional laptop within 45 seconds.

Afterwards the results can be plotted using Matplotlib. This includes graphical timetables, train-run profiles, energy consumption profiles, line profiles and station occupation charts.

In addition there are protocol files for the chosen train paths, the driving dynamics and the block occupation.

\subsection{Speed profiles}

The tool calculates the run in advance in order to get the shortest running time which is important for the calculation of the travel time reserve. Also all braking curves are calculated before the simulation run. The running speed is lower than the maximum speed according to the travel time allowance. At timetable points the delay is calculated and if necessary, the train travels at maximum speed in order to compensate for the time lost.

\subsection{Signalling system}

The distance keeping procedure is based on fixed blocks. These blocks can have a widespread length. It is also possible to shorten it for a high-capacity block system. The block length can be shorter than the train and also the corresponding braking curves can be overlapping at one point. Thus it is easy to handle high-speed traffic and other special situations in railway operation with this system. The traditional national signalling systems are not modelled exactly, but the results of the implemented system are close to reality.

\subsection{Coupling and decoupling procedure}

The virtual coupling is an overlay to the conventional signalling system, requiring the following train to ignore the signals.

In the timetable file there is an instruction for a train to follow a specific other train. It is not specified whether the so-called 'follow run' (FR) reaches the state of virtual coupling or the distance between the trains is larger (up to one kilometre). The following train is always stopping and waiting at the beginning. The first train, which is called the 'leading train', passes the common switch at the beginning of the common track and initiates the FR. The following train then enters the occupied block and is self-responsible for keeping the relative braking distance to the leading train. In the simulation this is not that difficult because all exact position and speed data is available every time and everywhere. In real application very fast and accurate technical systems for localization and communication would be required.

In the simulation the two trains are running on a line with a conventional signalling system with fixed blocks. The leading train initiates the block occupation and the following train initiates the block release. Both trains know their dividing switch with the surrounding signal blocks. The following train observes the dividing switch and keeps the absolute braking distance to that switch as long as the switch is not cleared by the leading train. After splitting the trains are following the rules of the conventional signalling system on their own and the FR is finished. 


\section{SHINKANSEN SCENARIO}

4.1 Tokaido line and current timetable

The Tokaido line was the first Shinkansen line in Japan opened in 1964. The distance from Tokio to Osaka is $515 \mathrm{~km}$ and the operational speed is $285 \mathrm{~km} / \mathrm{h}$ since 2015 (the simulation still uses the previous maximum speed of $270 \mathrm{~km} / \mathrm{h}$ ) [7]. Besides the cities Tokio, Nagoya, Kyoto and Shin-Osaka, the fastest train, called 'Nozomi', also stops at the stations Shinagawa and Shin-Yokohama in the Tokio area. The 'Hikari' trains have even more stops and the 'Kodama' trains stop at each of the 17 stations (see Fig. 4). The train density is very high. From Tokio there are 11 trains departing in the peak hour. The Nozomi trains depart every 10 min. Furthermore 2 Hikari and 2-3 Kodama trains depart within one hour from Tokio.

At smaller stations almost every stopping train is overtaken by faster trains, sometimes even by two. Especially the 'Atami' station is very challenging since, in contrast to all other small stations, there are no passing loops. The platform is situated directly at the high-speed track. Thus stopping trains are blocking the whole line for a certain time.

For the simulation only trains of the type Shinkansen 700 are used. Every train has the maximum length of $405 \mathrm{~m}$, either as 16-car train or with 8-car trains in double traction. With this the platform length is fully used. The weight of the trains is 708 tons, the acceleration reaches $0.9 \mathrm{~m} / \mathrm{s}^{2}$ and there are 1323 seats available.

The Kodama trains have the highest energy consumption due to the frequent stops $(22 \mathrm{kWh} / \mathrm{km}$, without recovery); the Nozomi trains are consuming $16 \mathrm{kWh} / \mathrm{km}$ (Fig. 5).

\subsection{Stations Tokio and Nagoya}

Stations often are the bottleneck in railway networks. Also the Shinkansen stations are designed quite minimalistic.

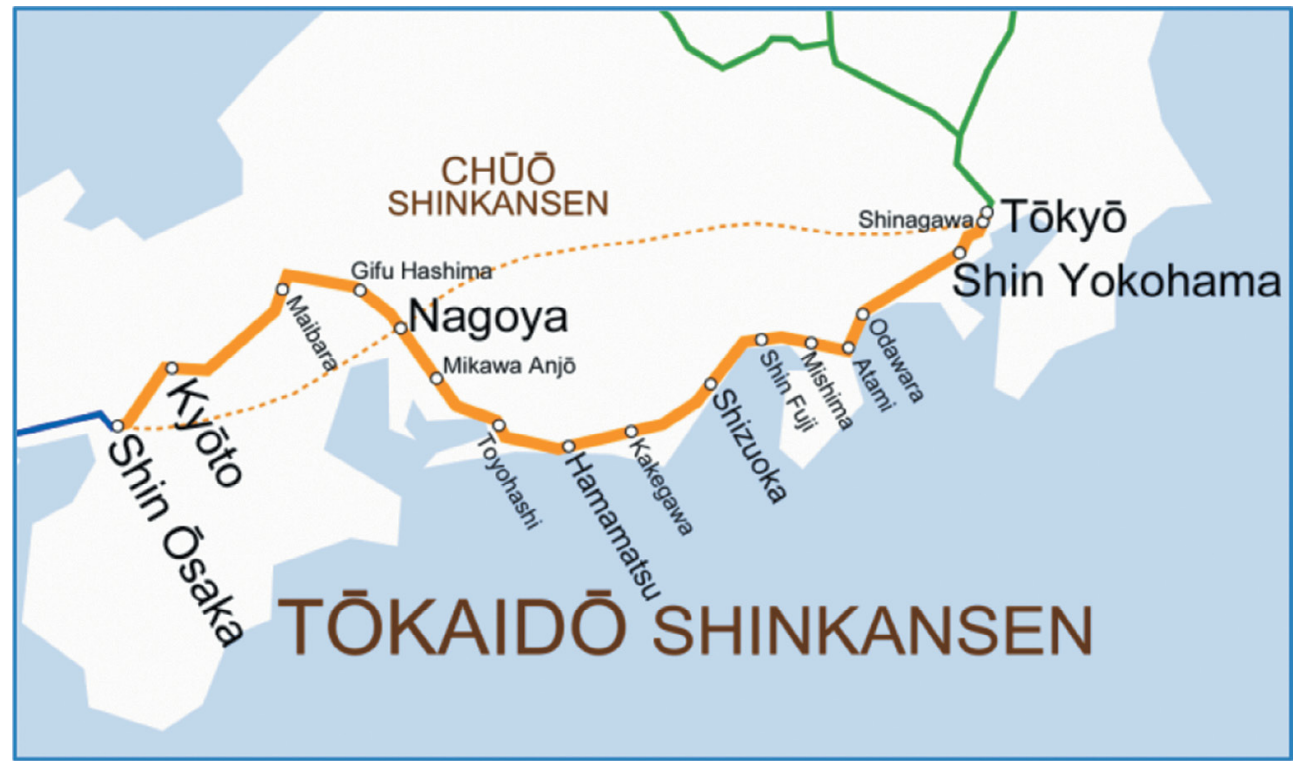

Figure 4: Alignment of Tokaido Shinkansen line (source: Hisagi/Wikipedia). 


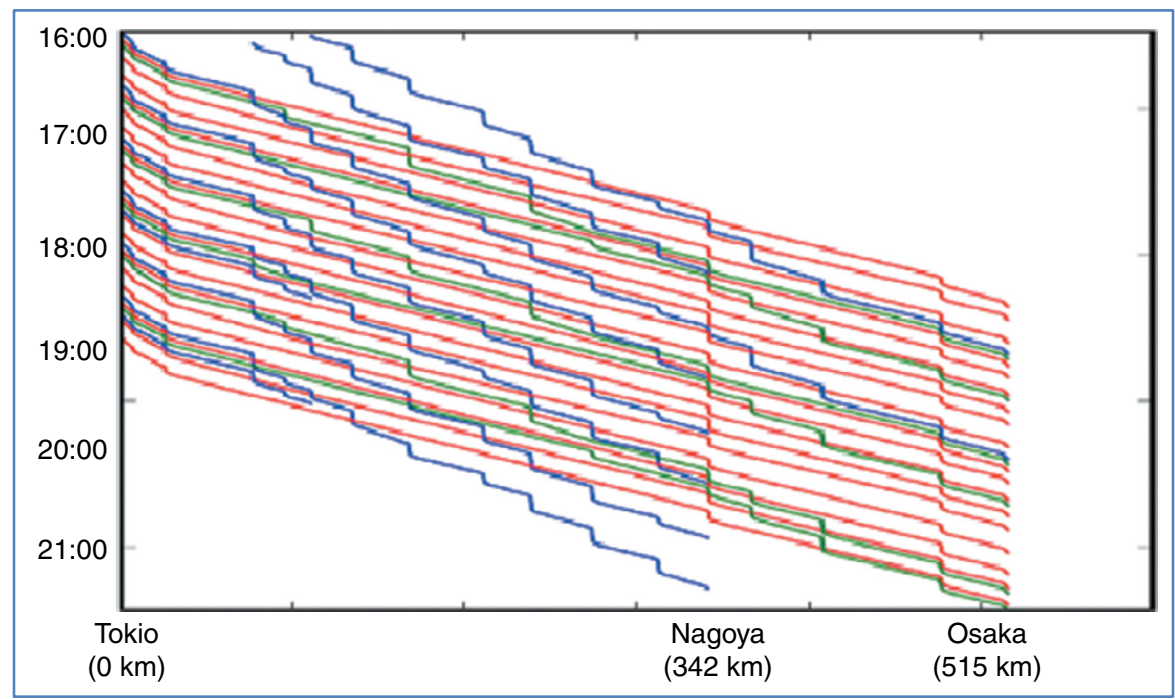

Figure 5: Graphical timetable for the Tokaido line including westward departing trains between 16:00 and 19:00, the fast Nozomi in red, the stopping Kodama in blue.

The Tokaido part of the Tokio station consists of six platform tracks only with no connection to other tracks of the station (see Fig. 6). The trains arrive at the switch area, where they block the tracks for departing train in half of the cases. Then they arrive at the platform quite slowly because of the track ending. After the passengers have left the train, the train undergoes a short cleaning, the seats are rotated and the other passengers enter the train. Every task takes a couple of minutes. The passenger change rate (number of embarking and disembarking passengers compared to the seat capacity) is very high. Thus it is challenging to increase the number of departing trains, which produces the benefit of virtual coupling, without changing the station infrastructure.

Nagoya has only four Shinkansen tracks with two island platforms. The sidetracks for stabling can only be reached from two tracks. So the track for ending and departing trains in Nagoya is fixed. It can be shown that the current infrastructure in Nagoya is insufficient for a noteworthy increase of train numbers.

\subsection{Simulation}

The timetables for the Tokaido line are taken from JR Central for the year 2015 [8]. In the peak hour 11 westbound trains are departing from Tokio. In the morning these are 10 and at noon 8 trains per hour. All departing trains from 4 p.m. to 7 p.m. are included in the simulation. Additionally the terminating trains are modelled to evaluate the situation in the Tokio station both at the platform tracks and the switch area.

\subsubsection{Doubling of Nozomi trains (DF1)}

The first scenario called DF1 is the virtual coupling of the Nozomi trains. All Nozomi trains get a double which is following them using the FR procedure. The design of the stopping stations allows the doubling of all Nozomi trains. Besides the main stations also the smaller stations have two platform tracks per direction which allows for parallel stopping. So there 


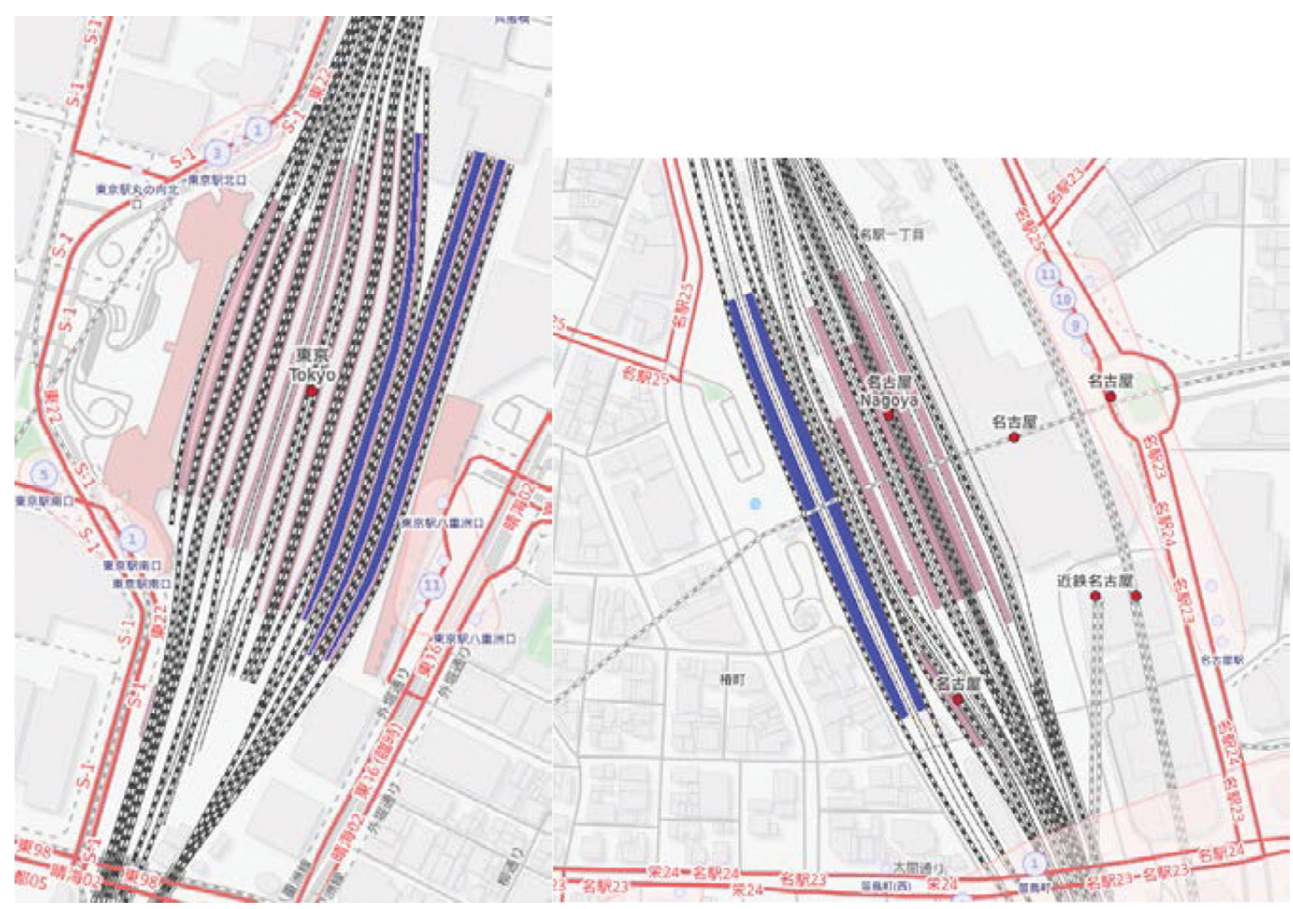

Figure 6: Tokio and Nagoya Station Layout - blue platforms belong to Tokaido line (Base Map: Transport by OpenStreetMap).

are always two 400-meter trains stopping at the stations. The following train arrives shortly after the leading train. There is no possibility for overtaking at the stopping stations. Hikari and Kodama trains have to stop at the smaller stations in the passing loop while the Nozomi pair of trains overtakes on the main track.

The simulation reveals the resulting distances between the trains during FR which vary from $900 \mathrm{~m}$ to a few kilometres. In case of disturbances the distance shortens to a few meters.

As expected one bottleneck is occurring in this scenario since the Nagoya station is not capable to handle the number of trains for the following reasons:

- The dwell time is $3 \mathrm{~min}$; whereas a Nozomi pair of trains blocks the station for more than 5 min in one direction.

- There are sometimes both a Hikari and a Kodama placed between two original Nozomi slots.

- Terminating Kodama trains also stop for 3 min to ensure a complete disembarking of all passengers.

Besides this resulting bottleneck the scenario generates a big benefit. The number of departing trains from Tokio is increased from 34 to 52 within the three hours. The passenger capacity increases from 15,000 to 23,000 passengers per hour and direction (Figs 7 and 8).

\subsubsection{Optimized scenario (DF1opt)}

To reduce the bottlenecks and delays which are not compatible with the self-conception of Shinkansen which stands for a punctual and reliable traffic mode, the scenario is optimized. The so-called DF1opt scenario differs in the following aspects: 

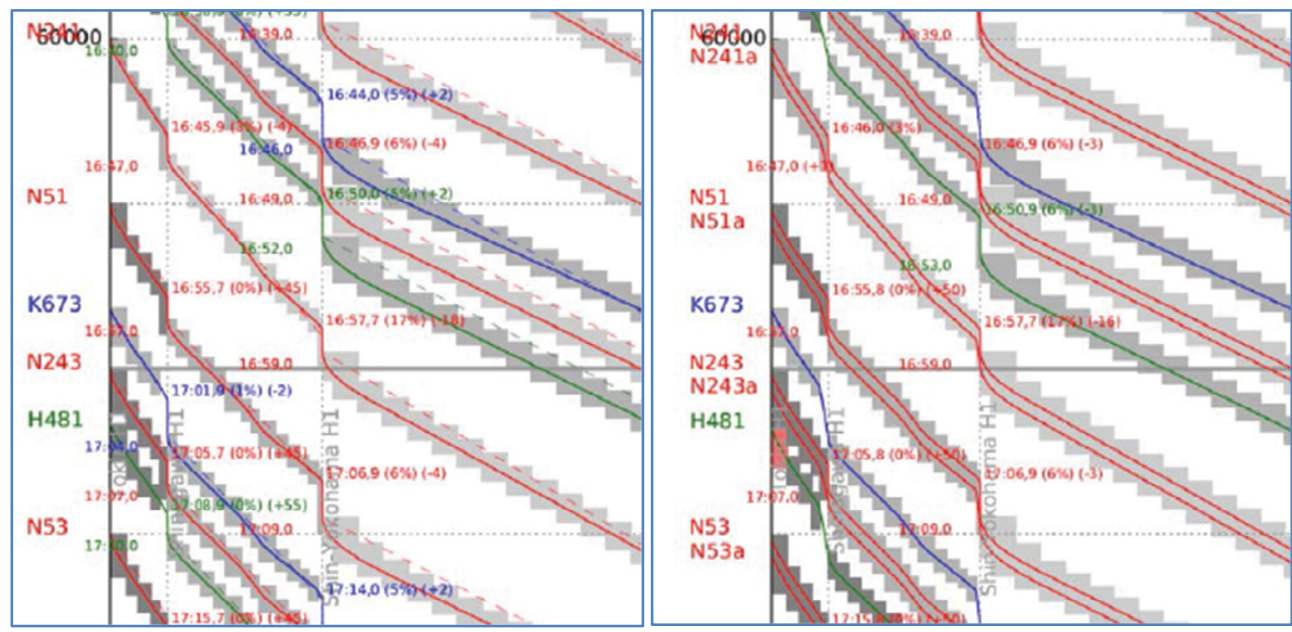

Figure 7: Graphical timetable for the Tokio area - original (left), DF1 Nozomi 'doubling' (right).

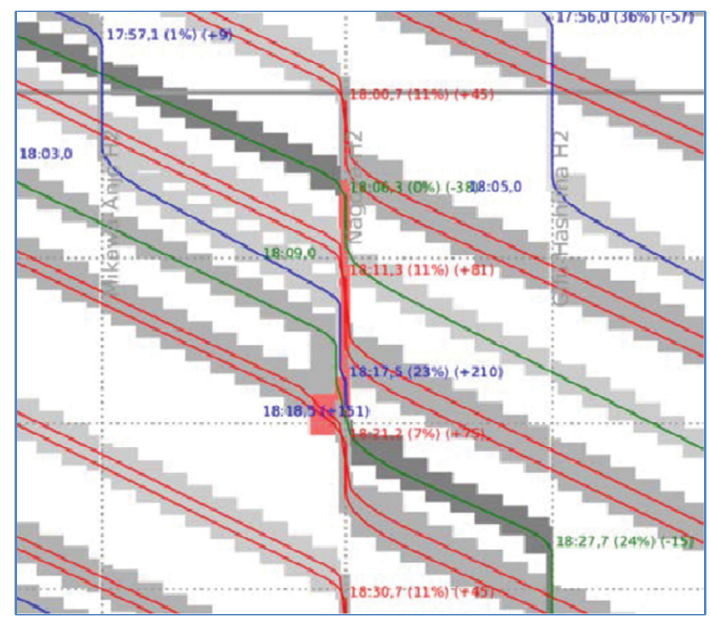

Figure 8: Graphical timetable Nagoya Bottleneck - red rectangles represent rejected track occupations, the numbers in brackets the delay in seconds.

- Reduction of the dwell time in Nagoya to 2 min

- Shift of departure times and train slots to eliminate conflicts

- Reduction of stopping times for the overtaken trains and moving them into the running time.

With these adjustments the delays are reduced but not eliminated completely. It is questionable whether it is realistic to shorten the dwell times in the main stations in such a way.

It can be noted here that Nagoya in any case needs at least one new platform track per direction.

4.3.3 Virtual coupling for travel time reduction (DF2)

This scenario aims to reduce the travel time of the Hikari and Kodama trains. These trains spend a lot of time waiting at the small stations to be overtaken by non-stop trains. The goal 


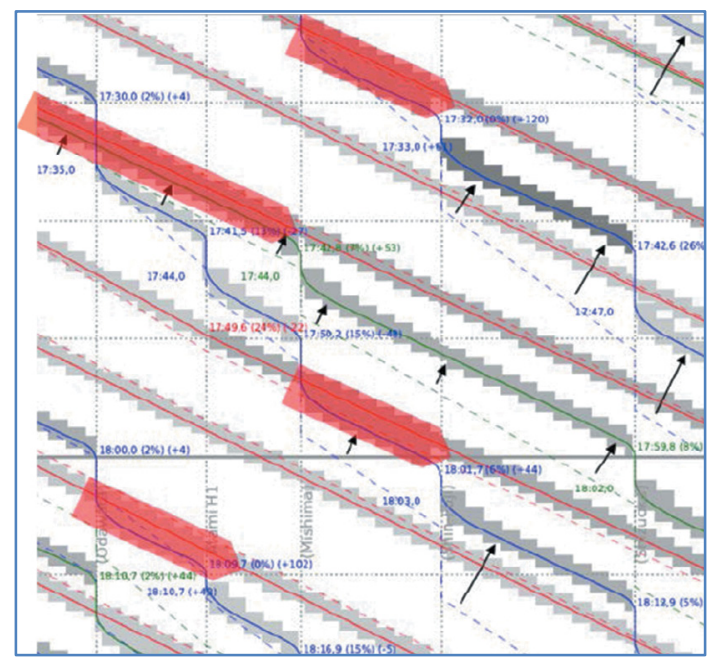

Figure 9: Scenario DF2 with a more compact timetable to create gaps for new trains (dark rectangles represent block occupations with less or no time allowance.

is to introduce the FR for the non-stop trains if there are two of them. This case occurs if a Hikari follows a Nozomi or the other way round. Additionally the FR shall be used by Kodama trains following their overtaking faster trains (see Fig. 9). Possibly the Kodama train is able to cut the time so that it must not be overtaken at the next station, but one station further.

A third goal is the insertion of new trains into gaps which possibly occur in the timetable due to the reduction of stopping times.

The buffer time between two trains slots must not fall below $1 \mathrm{~min}$ and in case of an overtake 2 min respectively. The running time supplement must not be less than $3 \%$, for the long sections of Nozomi trains at least $7 \%$.

Unfortunately this scenario fails to meet the expectations for the following reasons:

- Hikari trains stop quite often, which generates gaps that cannot be filled.

- Atami station is very challenging due to the missing passing tracks.

- Nagoya station is a bottleneck which compensates the benefit of a virtual coupling of Hikari and Nozomi.

\section{CONCLUSION}

For the virtual coupling there are several operational scenarios possible that promise benefit. Two main scenarios have to be regarded: the use of conventional and passive switches.

The first scenario of virtual coupling with conventional switches on the Japanese Shinkansen high-speed line shows the big benefit of adding trains that follow a leading train almost virtually coupled. These trains depart shortly one after another and arrive in the same way at the next station where two platform tracks are necessary. If two 400-metre Shinkansen trains follow each other the capacity of the line could be increased from 15,000 to 23,000 passengers per hour and direction. The simulation of the scenario reveals bottlenecks at some stations, especially in Nagoya with only two tracks per direction. 
With the DLR simulation tool DFSimu, which is able to handle virtual coupling, some more scenarios will be created, simulated and analysed. One of these will be the Slip Coaching Scenario for European high-speed lines.

\section{REFERENCES}

[1] Next Generation Train on the DLR Homepage, available at http://www.dlr.de/dlr/en/ desktopdefault.aspx/tabid-10467/740_read-916/\#/gallery/2043 (accessed 3 June 2016).

[2] Pachl, J. Systemtechnik des Schienenverkehrs, 7th ed., Springer Vieweg: Wiesbaden, pp. 35-36

[3] Slip Coaches, Dividing Trains at Speed, available at http://mikes.railhistory.railfan.net/ r134.html (accessed 3 June 2016).

[4] OpenTrack, available at http://www.opentrack.ch/ (accessed 3 June 2016).

[5] RailSys, available at http://www.rmcon.de/railsys-en/workflow/ (accessed 3 June 2016).

[6] FBS, available at http://www.irfp.de/english/fbs/ (accessed 3 June 2016).

[7] Andersen, S., Hochgeschwindigkeitsland Japan im Jahr 2015. Eisenbahn-Revue International, Minirex AG: Luzern, pp. 492-494, October 2015.

[8] Timetable of Tokaido Shinkansen on the website of Central Japan Railway Company, available at http://english.jr-central.co.jp/info/timetable/ (accessed 3 June 2016). 